\title{
A Novel Methodology for Bio-electrospraying Mesenchymal Stem Cells that Maintains Differentiation, Immunomodulatory and Pro-reparative Functions
}

\author{
${\text { Zita } \text { McCrea }^{1} \cdot \text { Yonsuang Arnanthigo }^{1} \cdot \text { Sally-Ann Cryan }}^{2,3,4,5} \cdot$ Shirley O’'Dea $^{1,6}$
}

Received: 6 October 2016/Accepted: 19 June 2017/Published online: 5 October 2017

(C) Taiwanese Society of Biomedical Engineering 2017

\begin{abstract}
Mesenchymal stem cells (MSCs) are an important cell source for tissue engineering (TE) and cell therapies for several reasons including ease of isolation from multiple tissues, uncomplicated ex vivo culture, ability to self-renew and differentiate into numerous cell types, MSC/immune cell interactions and pro-reparative properties. Current MSC therapies involve administration via intravenous (I.V.) injection. However, this can result in MSC entrapment and failure to target injured site. In TE, artificial 3D constructs are being investigated as strategies for direct delivery of MSCs to a target area. However, these constructs have numerous limitations including lack of cell infiltration, poor cell functionality and limited diffusion of nutrients and oxygen through the scaffolds. We are investigating the jetting methodology bio-electrospraying (BES) as an alternative strategy for MSCs delivery in vivo that may overcome obstacles associated with I.V. injections and scaffold transplantation. For BES in vivo, low voltages, stable jetting and a single needle configuration are highly
\end{abstract}

Shirley O'Dea

shirley.odea@nuim.ie

1 Biology Department, Maynooth University, Maynooth, Ireland

2 Tissue Engineering Research Group, Department of Anatomy, Royal College of Surgeons in Ireland, 123, St. Stephens Green, Dublin 2, Ireland

3 Trinity Centre for Bioengineering, Trinity College Dublin, Dublin 2, Ireland

4 School of Pharmacy, Royal College of Surgeons in Ireland, 123, St. Stephens Green, Dublin 2, Ireland

5 Centre for Research in Medical Devices (CÚRAM), National University of Ireland Galway, University Rd, Galway, Ireland

6 Avectas, Dublin, Ireland desirable. A commercially available electrospray apparatus Spraybase ${ }^{\circledR}$ was used to electrospray mouse bone marrowderived MSCs (mBMSCs) at low voltages $(\sim 3-6 \mathrm{kV})$ in vitro. Stable jetting conditions with a single needle at these low voltages were established by employing a ringshape electrode for potential difference, specific culture medium and the use of high mBMSCs numbers to overcome viscosity difficulties. The viability and functionality of the mBMSCs following BES was determined by analysing expression of specific surface markers, multilineage differentiation, suppression of T- cell activation and proreparative capabilities. We show that $\mathrm{mBMSCs}$ post-BES functioned similarly to non-bio-electrospray (non-BES) control mBMSCs for all parameters examined. This methodology may subsequently enable targeted delivery of MSCs to an injury site in vivo and potentially avoid the complications associated with MSCs entrapment and the limitations associated with artificial scaffolds.

Keywords Bio-electrospraying · Mesenchymal stromal/ stem cells · Cell delivery · Tissue engineering · Cell therapies $\cdot$ Bone marrow stem cells

\section{Introduction}

Mesenchymal stem cells, also called mesenchymal stromal cells (MSCs) are an important cell source for tissue engineering (TE) and cell therapies. They are readily accessible from multiple tissues, including bone marrow and adipose tissue. MSCs have ability to self-renew and differentiate into numerous cell types including; osteoblasts, chondrocytes, myocytes, adipocytes and fibroblasts [1]. These cells are also easily isolated and cultured ex vivo. Human MSCs (hMSCs) can be isolated from the bone marrow of patients 
and as such are a clinically viable cell source. Importantly, hMSCs can be directly implanted back to injured regions in the same patient, significantly reducing the possibility of transplant rejection [2]. In addition to this, MSCs are also favoured for therapies due to their interactions with immune cells and their expression of low levels of major histocompatibility complex (MHC) class I, and no MHC class II which allows them to evade allogeneic reactions and natural killer cell-mediated killing [3].

Patient MSC therapy currently involves administration through intravenous (I.V.) injection. This allows cells to move through the blood stream to any site in the body, regardless of the intended target site and the efficiency of targeting the injury site can be low. Furthermore, a welldocumented phenomenon, MSC entrapment, can occur after MSCs are administrated by I.V. injection whereby MSCs remain in the lungs and liver of rodents [4]. This has also been reported in patient trials [5-10]. The risk of acute or chronic adverse side effects from entrapment of cells in the lungs or liver means that careful patient monitoring is essential.

In tissue engineering, conventional strategies for administrating MSCs to a target area include the fabrication of artificial 3D constructs. These constructs should mimic the structure and function of the native tissue while facilitating regeneration in vivo [11]. However, to date, the synthetic environment of these 3D constructs has numerous limitations including lack of cell infiltration through the scaffold, limited diffusion of oxygen and nutrients and failure of desired cell functions. This is generally due to small pore sizes, the chemistry of the materials used to fabricate the scaffolds and the surface texture of the scaffolds. Numerous reviews have discussed the wide range of studies carried out to address these problems, however the fabrication of successful in vivo 3D constructs remains challenging [12-17].

In 2006, the Jayasinghe group was the first to report the adaptation of a well-established physics phenomenon called electrospraying for cell delivery and TE applications. Briefly, electrospraying is a jetting methodology whereby tiny quantities of electrical charge are applied to a fluid as it passes through an emitter resulting in the formation of a spray jet. This spray is finer, more controllable and significantly faster than aerosol [18]. By electrospraying a solution of living cells with no apparent deleterious effect on the cells, the group developed the process known as 'bio-electrospraying' (BES) [19-22]. Since then, a range of cell types have been reported as being successfully electrosprayed including primary cardiac cells (PCCs), mouse hematopoietic stem cells (mHSCs), mouse bone marrow derived stem cells (mBMSCs), embryonic stem cells (ESCs) and human adipose derived stem cells (hASCs) [23-28]. In establishing stable jetting conditions for BES, parameters such as potential difference, flow rate of cell/culture medium solution and culture medium properties are important [29-31]. Potential difference refers to the difference in potential between the emitter and the ground electrode. Three types of ground electrodes have been employed in studies aiming to achieve stable cone-jets: ring, plate and point-shaped electrodes. The properties of the culture medium, including viscosity, electrical conductivity, surface tension and density, as well as the consistency of the flow rate of culture medium through the needle are also critical parameters for generation of stable jetting conditions [32]. Viscosity and electrical conductivity are two significant parameters that can affect jetting stability. Low viscosity can lead to unstable jetting. Previous studies addressed the problem of low viscosity by electrospraying solutions containing high numbers of cells, which results in increased viscosity levels $[24,28]$. However, this also results in increasing already high conductivity levels, which leads to unstable jetting with existing electrospray apparatuses. To address this, groups have used coaxial needles and a sheath gas to protect the cells and prevent sparking at the high voltages that are necessary to produce stable jets. This configuration is not compatible with in vivo applications. Efforts to use a single needle rather than a coaxial needle have not been successful to date [32].

We propose that BES may be an important strategy for delivery of MSCs to specific injury sites in vivo. However, studies carried out to date are not compatible with in vivo use because of the high voltages required to generate stable cone jets. High voltages are not desirable clinically and lead to MSC death and loss of function. While other groups have used electrospray apparatuses assembled inhouse, we used Spraybase $^{\circledR}$, a commercially available electrospray instrument which facilitates consistent electrospraying and requires lower voltages than in-house assemblies. We also optimised specific parameters to achieve a stable BES using a lower and a single needle rather than higher voltages, a coaxial needle and a sheath gas. Furthermore, previous studies have not comprehensively examined the functionality of MSCs post-BES. Here we have examined tri-lineage differentiation, expression of specific surface markers associated with MSCs, suppression of T- cell activation and pro-reparative properties of BES, and we demonstrate that mBMSCs behaved similarly to their non-bio-electrosprayed (non-BES) counterparts in all biological aspects, potentially providing an alternative delivery method for MSC therapies, and tissue engineering and regenerative medicine purposes. 


\section{Materials and Methods}

\subsection{Animals and Cells}

Six-to-eight week old female BALB/c mice and C57/BL6 mice (Charles River Lab Manston Rd, Margate, UK) were used for experiments under the guidelines with ethical approval received from the ethics committee of Maynooth University (Ref: BSRESC-2012-015). The A549 human lung adenocarcinoma cell line and human bronchial epithelial cell line (BEAS 2B cells) were obtained from the European Collection of Cell Cultures.

\subsubsection{Isolation and Culture of Murine Bone Marrow- Derived MSC}

Murine BMSCs were isolated and expanded using modifications of methods previously described [33, 34]. Modifications included flushing out bone marrow using specialised mesenchymal stem cell medium 'Mesenchymstem' (GE Healthcare Life Sciences, Buckinghamshire, UK). Isolated cells $\left(6.0-6.5 \times 10^{7}\right)$ were suspended in Mesenchymstem containing $10 \mu \mathrm{g} / \mathrm{ml}$ penicillin/streptomycin (P/S) (Gibco, Paisley, UK) in T75 flasks and incubated in hypoxic conditions $\left(5 \% \mathrm{O}_{2}\right)$. Non-adherent cells were removed after $3 \mathrm{~h}$. The flasks were washed with sterile PBS after 24 and $48 \mathrm{~h}$ and incubated for 3 weeks until colonies of fibroblast-like cells appeared. Cells were passaged by trypsinisation with $0.2 \mathrm{mM}$ trypsin/ $1 \mathrm{mM}$ EDTA (Invitrogen-Gibco) for $2 \mathrm{~min}$ at $37{ }^{\circ} \mathrm{C}$. Cells were centrifuged $(400 \times g, 5 \mathrm{~min})$ and re-seeded at $5-6 \times 10^{6}$ cells in T75 (passage 1). Passage 2 cells were cultured in mesenchymstem media, $10 \mu \mathrm{g} / \mathrm{ml}$ penicillin/streptomycin and $1 \mathrm{ng} / \mathrm{ml}$ murine basic fibroblast growth factor (bFGF) (Peprotech, Rocky Hill, NJ, US). Passage 3 cells were cultured in expansion medium (c $\alpha$-MEM medium) consisting of Minimum Essential Medium Alpha medium (Gibco) supplemented with $10 \%(\mathrm{v} / \mathrm{v})$ heat inactivated fetal bovine serum (FBS) (Gibco), 10\% (v/v) equine serum (Hyclone laboratories, Logan, UT), $10 \mu \mathrm{g} / \mathrm{ml}$ penicillin/ streptomycin, $10 \mu \mathrm{g} / \mathrm{ml}$ L-glutamine (Gibco) and $1 \mathrm{ng} / \mathrm{ml}$ murine bFGF in T-175 flasks. Cells were maintained in either hypoxic $\left(5 \% \mathrm{O}_{2}\right)$ (passage $\left.1-3\right)$ or normoxic $(21 \%$ $\mathrm{O}_{2}$ ) (passage 4-10) conditions. Surface marker expression, multilineage differentiation and $\mathrm{T}$ cell suppression assay was carried out as described below to ensure that mBMSCs were not contaminated with hematopoietic or other cell contaminants, and that cells retained differentiation capacity as previously described [34]. Cells were used between passages $3-10$.

\subsection{Bio-Electrospray Apparatus and Optimization}

The 'Generation 1' BES instrument was purchased from Avectas (Dublin, Ireland) and consisted of a voltage supply, double syringe pump, a single extrusion needle (30 gauge) configuration, lab jack, camera, laser and ringshaped ground electrode. Working in a dark, non-sterile environment, mBMSCs ranging from $1 \times 10^{6}$ and $6 \times 10^{6}$ cells $/ \mathrm{ml}$, suspended in c $\alpha$-MEM medium, were delivered using the syringe pump via a $1 \mathrm{ml}$ syringe to the extrusion needle. The conductivity of the $\mathrm{mBMSCs} / \mathrm{c} \alpha$ MEM medium solution was measured as $22.18 \mathrm{mS} / \mathrm{cm}$ using a conductivity metre (Mettler Toledo, Ireland). An electric field was applied to the needle to draw the cells into jets and deposit them in droplets into 6-well or 24-well sterile tissue culture plates. Sprays were illuminated with the laser and photographs were taken with the camera 1 frame/sec over $5 \mathrm{~s}$ to determine when a continuous, stable Taylor cone was achieved. Voltages of 1, 2, 3, 4, 5 and $6 \mathrm{kV}$ were tested and distances and flow rates were varied. It was established the optimum conditions for electrospraying mBMSCs with this device used voltages between 3 and $6 \mathrm{kV}$, which varied depending on the relatively humidity (RH), flow rate at $5 \mu \mathrm{l} / \mathrm{min}$ and potential distance of $22 \mathrm{~mm}$. 24-well plates were used to collect cells for studies examining mBMSCs viability and proreparative properties while mBMSCs were collected in 6-well plates for differentiation and immunomodulatory studies.

\subsection{Measurement of Consistency of Bio- Electrospray Delivery}

A suspension of $3.5 \times 10^{6} \mathrm{mBMSCs}$ in $300 \mu \mathrm{lc} \alpha$-MEM medium was bio-electrosprayed at a flow rate of $5 \mu \mathrm{l} / \mathrm{min}$. Three $100 \mu \mathrm{l}$ fractions were collected sequentially in three separate wells of a 24 well plate. These cells were immediately harvested and counted using a haemocytometer (Sigma-Aldrich) and Olympus CK40 Light Microscope (Olympus, Germany).

\subsection{Viability of mBMSCs}

On three separate days, $1 \times 10^{6} \mathrm{mBMSCs} / \mathrm{ml}$ were electrosprayed into three wells. The cells were immediately harvested from the wells and counted using a haemocytometer and ethidium bromide/acridine orange (EB/AO) viable staining. In addition, $1 \times 10^{6}$ pipette mBMSCs, which represent non-BES cells, and BES mBMSCs were harvested and suspended in $500 \mu \mathrm{l}$ PBS supplemented with $1 \%(\mathrm{v} / \mathrm{v})$ FBS (FACS buffer). Furthermore, $5 \mu \mathrm{l}$ of 7-amino-actinomycin D (7-AAD) Staining Solution (eBioscience, San Diego, CA, US) was added, the cells were 
incubated for $15 \mathrm{~min}$ at $4{ }^{\circ} \mathrm{C}$ and then analysed by flow cytometry (FACS Accuri, California, USA) using CFlowPlus software (BD Biosciences, Oxford, UK) on day 1, 7, 14.

\subsection{Surface Marker Detection on mBMSCs}

Both pipette and BES mBMSCs were harvested, washed in sterile PBS and suspended in FACS buffer to yield approximately $1 \times 10^{5}$ cells $/ 4 \mathrm{ml}$ polypropylene FACS tubes (Falcon, BD Biosciences). Fluorochrome conjugated antibodies (Table 1) and their isotype controls were incubated with cells for $15 \mathrm{~min}$ at $4{ }^{\circ} \mathrm{C}$. Cells were then washed in $2 \mathrm{ml} \mathrm{FACS}$ buffer, vortexed and centrifuged $(300 \times g$, $5 \mathrm{~min})$. The supernatant was removed and cells were resuspended in $200 \mathrm{ml}$ FACS buffer. Cells were then analysed by flow cytometry.

\subsection{Tri-Linage Differentiation of mBMSCs}

\subsubsection{Osteogenic Differentiation of mBMSCs}

mBMSCs were both pipetted and BES at a density $5 \times 10^{4}$ cells $/ \mathrm{ml}$ into a 6-well plate and incubated at $37{ }^{\circ} \mathrm{C}, 5 \%$ $\mathrm{CO}_{2}$. Once confluent, typically after 3-4 days, cells were incubated for 21 days in osteogenic differentiation medium containing $\alpha$-MEM, $1 \mathrm{mM}$ dexamethasone (SigmaAldrich, Dublin, Ireland), $20 \mathrm{mM} \quad \beta$-glycerolphosphate (Sigma-Aldrich), $50 \mu \mathrm{M}$ L-ascorbic acid-2-phosphate (Sigma-Aldrich), $50 \mathrm{ng} / \mathrm{ml}$ L-thyroxine sodium pentahydrate (Sigma-Aldrich). The medium was then removed and cells were fixed in $10 \%(\mathrm{v} / \mathrm{v})$ neutral buffered formalin for $20 \mathrm{~min}$ at room temperature. After several washes in PBS, cells were incubated for $20 \mathrm{~min}$ in $1 \mathrm{ml}$ of $1 \%(\mathrm{w} / \mathrm{v})$
Alizarin Red S Stain. Cells were then washed with $\mathrm{dH}_{2} \mathrm{O}$. For visualisation, $1 \mathrm{ml}$ of $\mathrm{dH}_{2} \mathrm{O}$ was added to each well and cells were examined under the microscope.

\subsubsection{Adipogenic Differentiation of mBMSCs}

Pipette and BES mBMSCs were seeded at a density $5 \times 10^{4}$ cells $/ \mathrm{ml}$ into a 6 -well plate. Once confluent, cells were incubated for 21 days in adipogenic differentiation medium consisting of $\alpha$-MEM, $5.0 \mu \mathrm{g} / \mathrm{ml}$ insulin in $0.1 \mathrm{~N}$ acetic acid (Sigma-Aldrich), $50 \mu \mathrm{M}$ indomethacin (SigmaAldrich), $1 \mu \mathrm{M}$ dexamethasone (Sigma-Aldrich), $0.5 \mu \mathrm{M}$ 3-isobutyl-1-methylxanthine (IBMx) in methanol (SigmaAldrich). Cells were fixed in $10 \%$ neutral buffered formalin for $20 \mathrm{~min}$ at room temperature, washed in PBS, stained with $1 \mathrm{ml}$ of $0.5 \%(\mathrm{w} / \mathrm{v})$ Oil Red O Isopropanol fat differentiation stain and examined using a microscope.

\subsubsection{Chondrogenic Differentiation of mBMSCs}

Pipette and BES mBMSCs were seeded at a density $2 \times 10^{5}$ cells/ml into a 24 -well plate, immediately harvested and centrifuged $(300 \times g, 5 \mathrm{~min})$ in a $15 \mathrm{ml}$ polypropylene tube. The pellet was cultured in $500 \mu \mathrm{l}$ chondrogenic medium and incubated in normoxic incubator. The chondrogenic medium consisted of $\alpha$-MEM, $100 \mathrm{nM}$ dexamethasone, $50 \mu \mathrm{g} / \mathrm{ml}$ ascorbic-acid-2-phosphate (Sigma, Dublin,Ireland), $40 \mu \mathrm{g} / \mathrm{ml}$ proline (Gibco), $1 \mathrm{mM}$ sodium pyruvate (1:99) ITS + supplement (Gibco), $10 \mathrm{ng} / \mathrm{ml}$ TGF- $\beta 3$ (Peprotech). Fresh medium was added every 3-4 days for 21 days, after which the pellet was washed with Dulbecco's PBS. Total RNA from cells growing in a $15 \mathrm{ml}$ tube was isolated using TRIZOL Reagent (Invitrogen-Life Technologies). RNA was DNase
Table 1 Antibodies for flow cytometry

\begin{tabular}{lllll}
\hline Application & Antibody & Fluorochrome & Isotype & Supplier \\
\hline Flow cytometry & CD 11b & FITC & Rat IgG2b & e-Bioscience \\
& CD 34 & FITC & Rat IgG2a & e-Bioscience \\
CD 44 & PE & Rat IgG2b & e-Bioscience \\
CD 45 & PE & Rat IgG2b & e-Bioscience \\
CD 73 & PE & Rat IgG1 & e-Bioscience \\
CD 86 & PE & Rat IgG2a & e-Bioscience \\
CD 90.2 & FITC & Rat IgG2b & e-Bioscience \\
CD 105 & PE & Rat IgG2a & e-Bioscience \\
CD 106 & FITC & Rat IgG2a & e-Bioscience \\
CD 117 & FITC & Rat IgG2b & e-Bioscience \\
Sca-1 & PE & Rat IgG2b & e-Bioscience \\
MHC class 1 & FITC & Mouse IgG2a & e-Bioscience \\
MHC class 11 & PE & Rat IgG2b & e-Bioscience \\
\hline
\end{tabular}

Table of fluorochrome conjugated antibodies and isotype controls used for detecting surface markers for mBMSCs characterisation 
treated (Invitrogen-Life Technologies) and reverse transcribed using Superscript ${ }^{\circledR}$ III Reverse Transcriptase (Invitrogen-Life Technologies). PCR products were separated by agarose gel electrophoresis and visualised using GelRed Nucleic Acid Stain (Biotium, Cambridge, UK) using Quantity One densitometry software version 4.4.1 (Biorad, Hemel Hempstead, Hertfordshire, UK). Target transcripts were amplified using primers for Collagen IIa and Aggrecan (Sigma) and GAPDH (Eurofins) (Table 2).

\subsection{T-Cell Suppression Assay}

Both pipette and BES mBMSCs were seeded at $1.5 \times 10^{4} /$ $\mathrm{ml}$ in a 96-well plate in $100 \mu \mathrm{l}$ of $\mathrm{c} \alpha$-MEM and incubated overnight. The next day, splenocytes were isolated from the spleens of two MHC mismatched mice. BALB/c mice splenocytes' were represented as Donor 1 (D1) and C57/ BL6 mice as Donor 2 (D2). The suspended pellets were stained with carboxyfluorescein diacetate succinimidyl ester (CFSE) Proliferation Kit (CellTrace ${ }^{\mathrm{TM}}$, Life Technologies, Dublin, Ireland) using a previously described method [35]. Briefly, splenocyte pellets suspended in $1 \mathrm{ml}$ RPMI 1640 (Invitrogen-Gibco) supplemented with 5\% FBS were placed in $15 \mathrm{ml}$ polypropylene tubes and laid horizontally. PBS was pipetted at $110 \mu \mathrm{l}$ at the top of the $15 \mathrm{ml}$ tube, ensuring the PBS and the pellet did not mix. Following this, $1.1 \mu \mathrm{l}$ of $5 \mathrm{mM}$ CFSE $\left(\right.$ CellTrace $^{\mathrm{TM}}$ ) was added to the PBS. The solutions were then quickly inverted and vortexed to mix the cells and CFSE together. The tube was covered with foil and incubated for $5 \mathrm{~min}$ at room temp. Furthermore, $10 \mathrm{ml} 20{ }^{\circ} \mathrm{C}$ PBS containing 5\% FBS was added and centrifuged $(300 \times g, 5 \mathrm{~min})$. The pellets were then suspended in $1 \mathrm{ml}$ RPMI 1640 supplemented with $10 \%$ (v/v) FBS, $10 \mu \mathrm{g} / \mathrm{ml}$ penicillin/streptomycin, $10 \mu \mathrm{g} / \mathrm{ml}$ L-glutamine. Splenocytes were seeded at $2 \times 10^{5} /$ well and incubated for $72 \mathrm{~h}$. On day 5 , splenocytes were transferred to 96 well V-bottom plates (Corning Inc., NY, USA), centrifuged $(950 \times g, 5 \mathrm{~min})$ and supernatant was discarded. Cells were washed with $100 \mu \mathrm{l}$ FACS buffer, centrifuged $(950 \times g, 5 \mathrm{~min})$ and supernatant was discarded. Then $0.3 \mu \mathrm{l}$ CD3-APC (e-Bioscience), $0.3 \mu \mathrm{l}$ 7-AAD and $2.4 \mu \mathrm{l}$ FACS buffer were added to each well and plates were incubated at $4{ }^{\circ} \mathrm{C}$ for $15 \mathrm{~min}$. Cells were washed again with $200 \mu \mathrm{l}$ FACS buffer, centrifuged $(950 \times g, 5 \mathrm{~min})$ and supernatant was removed. $100 \mu \mathrm{l}$ FACS buffer was added to each well and splenocytes were analysed by flow cytometry.

\subsection{Scratch Assay (Wound Closure Assay)}

A549 or BEAS-2B cells were seeded at $5 \times 10^{4}$ cells/well in culture medium (Dubeco's Modified Eagles Basal Medium (DMEM-F12) (Gibco, Paisley, UK), 5\% FBS, $2 \mathrm{mM}$ L-Glutamine) into the lower chambers of a 24-transwell plate. After 24 h, a P200 pipette tip was used to scratch a line through the confluent monolayer of A549 cells in each well to make a wound. Following this, either pipette mBMSCs, BES mBMSCs or control media was added to the transwell inserts. After $24 \mathrm{~h}$, the A549 or BEAS-2B cells were fixed with $500 \mu \mathrm{l}$ of $10 \%$ formalin at room temp for $20 \mathrm{~min}$ and then stained with $1 \%$ crystal violet dye (Sigma, Dublin, Ireland) at room temp for 20 min. Plates were washed five times with PBS and cells were examined using a microscope. The width of the wound was measured at the top, middle and bottom of each well and an average width was determined for each well, demonstrating the width of wound closure obtained.

\subsection{Statistical Analyses}

Statistical analysis was performed using GraphPad Prism $^{\mathrm{TM}}$ software (GraphPad, San Diego, CA). Paired t-test was used when statistical analysis was required between two experimental groups. One way ANOVA was used to test for statistical significance of differences when multiple experimental groups were compared with post hoc tukey. Data are presented as the \pm standard error of the mean (SEM). P-values of $\mathrm{p}<0.05(*), \mathrm{p}<0.01(* *)$ or $\mathrm{p}<0.001(* * *)$ were considered statistically significant.

Table 2 Primers

\begin{tabular}{lllll}
\hline Primer & Forward 5'-3 & Reverse $3^{\prime}-5^{\prime}$ & Size $(\mathrm{bp})$ & Anneal temp. $\left({ }^{\circ} \mathrm{C}\right)$ \\
\hline Collagen IIa & GCGATGACATTATCTGTGAAG & TATCTCTGATATCTCCAGGTTC & 150 & 58 \\
Aggrecan & CTACCTTGGAGATCCAGAAC & TGGAACACAATACCTTTCAC & 121 & 58 \\
GAPDH & GCACAGTCAAGGCCGAGAAT & GCCTTCTCCATGGTGGTGAA & 151 & 58 \\
\hline
\end{tabular}

Table of primers for chondrocyte differentiation 
Fig. 1 "Generation 1" Spraybase ${ }^{\circledR}$ developed by Avectas. a Device consists of a syringe pump which delivers cell suspensions to a single extrusion needle, charged by IEC standard approved power supply unit, with laser and camera. b Ground ring electrode in well of 24-well plate

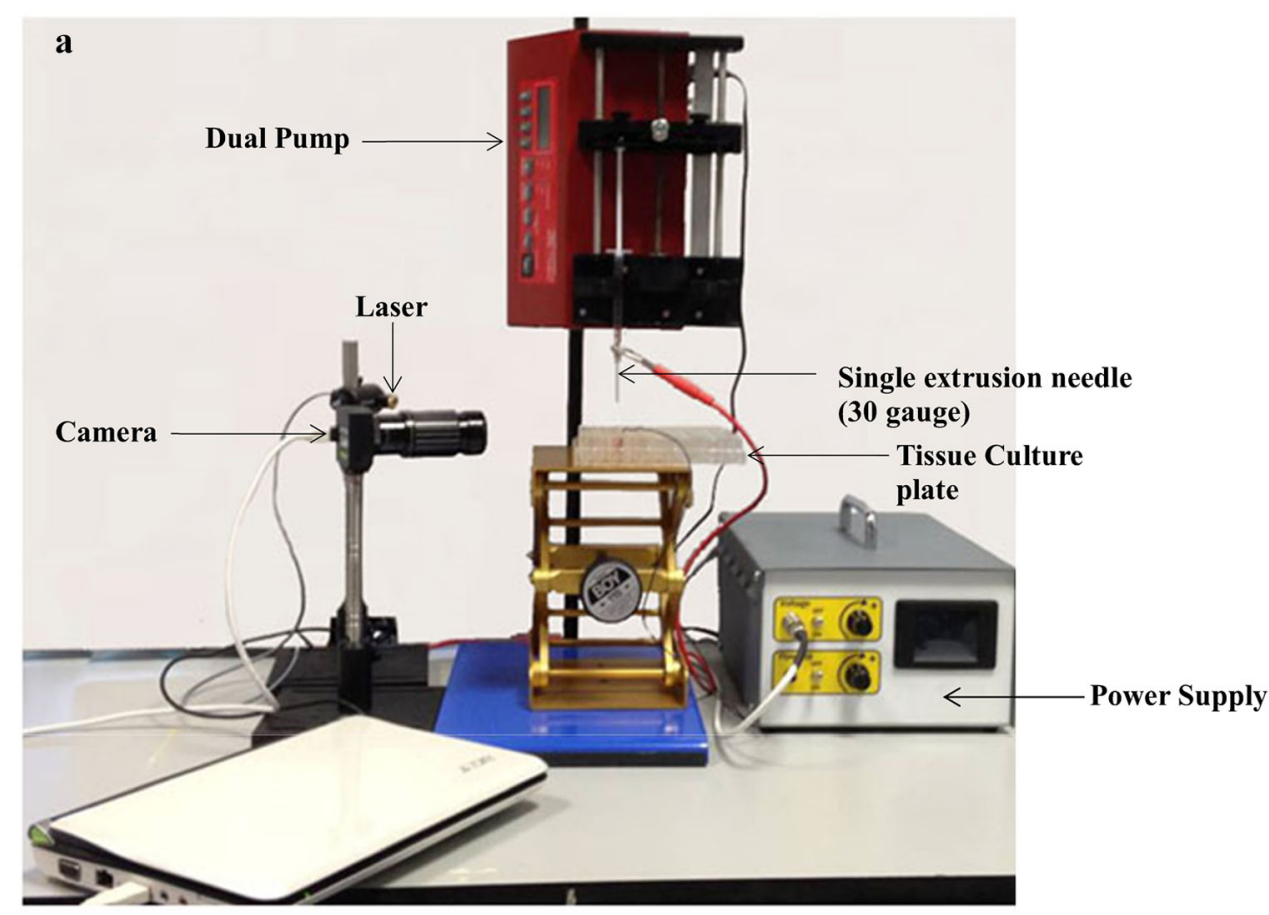

b
Ring-shaped electrode

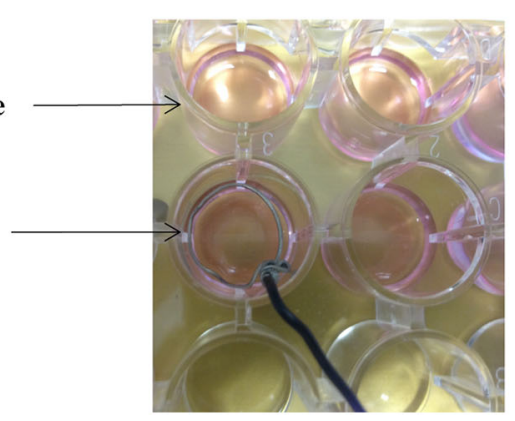

\section{Results}

\subsection{Optimization of Bio-Electrospray Parameters}

Previous studies have examined various parameters for successful BES of MSCs. The viscosity and electrical conductivity of the culture medium in which primary cells are sprayed have been shown to be important parameters that affect the ability to generate a stable jet. In previous studies, high cell numbers have been used to increase viscosity. However, this has resulted in high conductivity which in turn necessitates the use of voltages up to $15 \mathrm{kV}$ to generate stable jets. To avoid sparking at these voltage levels, a coaxial needle/sheath gas emitter configuration has been used. In the present study, we used a commercially available electrospray apparatus to optimise a range of parameters to develop a configuration that would allow successful generation of stable jets of mBMSCs at low voltages.
It is unclear from previous reports what exact apparatus was used for electrospray because the equipment appears usually to be built and assembled in-house. This also makes it difficult to reproduce results between labs. We used the newly commercially available Spraybase ${ }^{\circledR}$ Generation 1 instrument (Fig. 1a). We could achieve lower voltages with this instrument than with our previous in-house apparatus and we assume that this is due to the design of the Spraybase ${ }^{\circledR}$ instrument. Odenwalder et al. [32] used a ringshaped ground electrode which they placed into the culture well. The electrospray is attracted to the ground electrode and this shape is likely to encourage the cells to cover the bottom of the well. We used copper wire to form a similar ring-shaped electrode (Fig. 1b).

We discovered that cell suspensions of $1 \times 10^{6} \mathrm{cell} / \mathrm{ml}$ in c $\alpha$-MEM medium, a ring-shaped ground electrode, a single needle configuration and the purpose-built power supply contained within Spraybase ${ }^{\circledR}$ contributed to the successful generation of stable sprays/jets of mBMSCs at low voltages. A flowrate of $5 \mu \mathrm{l} / \mathrm{min}$ with voltages ranging 
Fig. 2 Electrosprays and jets. mBMSCs suspended in $\mathrm{c} \alpha$ MEM were BES at different voltages to get desired jet/spray. a, b show both jet and spray respectively. Optimum parameters include; $3-6 \mathrm{kV}$ (depending on $\mathrm{RH}$ ), flowrate of $5 \mu \mathrm{l} / \mathrm{min}$, potential distance $22 \mathrm{~mm}$, internal diameter of syringe $1.457 \mathrm{~mm}$ and 30 -gauge extrusion needle.

c Demonstrates an inconsistent and unstable BES intermittingly spurting out cells from the needle at $10 \mathrm{kV}$. Pictures were taken 1 frame/sec for 5 frames a Jet mode of mBMSC cell suspension with central ligament at $3-6 \mathrm{kV}$

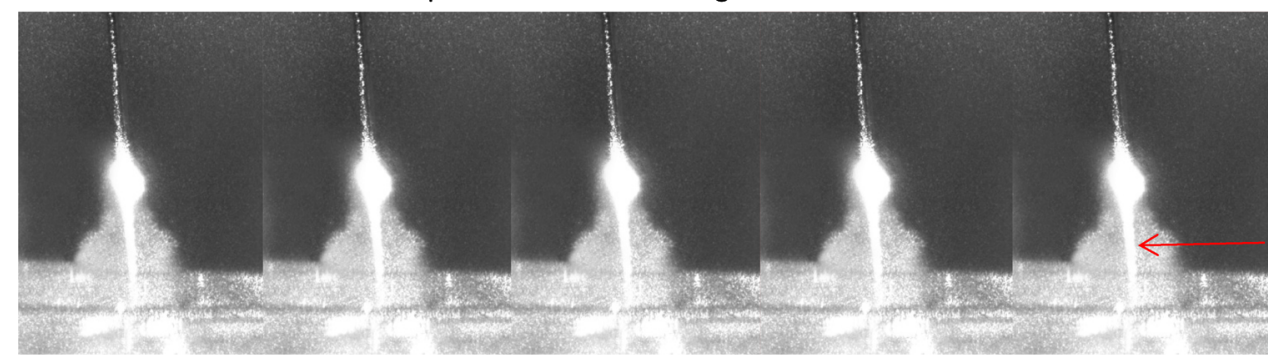

b Spray mode of mBMSC cell suspension at 3-6kV

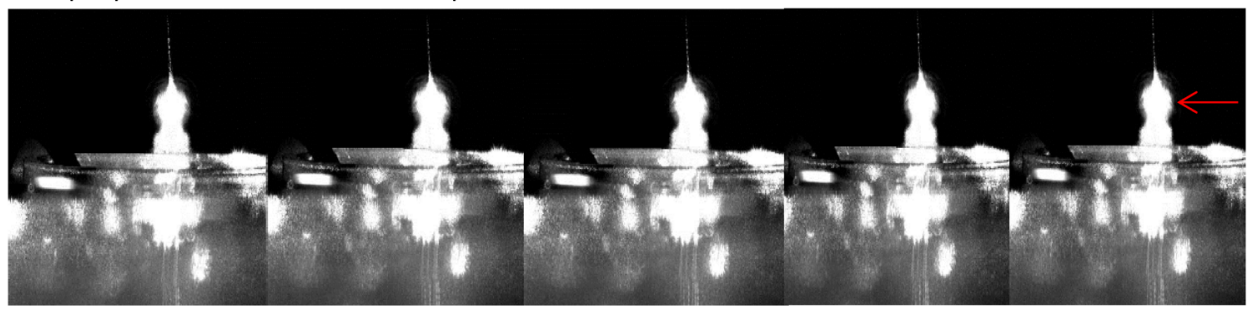

c Unstable jet/spray at $10 \mathrm{kV}$ intermittingly spurting out mBMSC cells

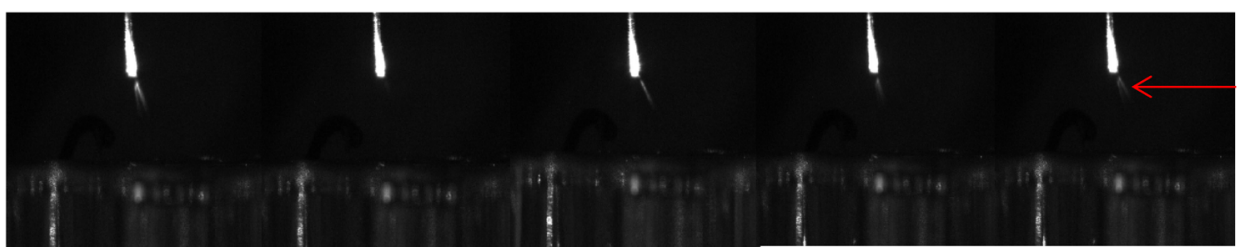

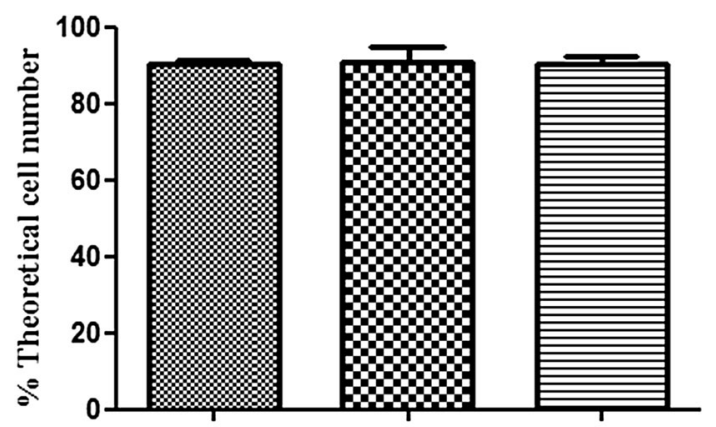

Fraction $1 \quad$ Fraction 2 Fraction 3

Fig. 3 Graph of distributed Bio-electrosprayed mBMSCs by fractions. Using optimum parameters, $3.5 \times 10^{6} / 300 \mu 1 \mathrm{mBMSCs}$ were electrosprayed into 3 separate wells of a 24 well plate (n-3). Using 3 fractions, cells were distributed evenly/100 $\mu$ l. These cells were then counted immediately post BES. The graph represents the number of cells counted in each well/100 $\mu \mathrm{l}$, demonstrating a continuous and even amount of cell suspension is distributed from the needle across the wells

from 3 to $6 \mathrm{kV}$, depending on the $\mathrm{RH}$, and a 30-gauge single needle configuration was subsequently used throughout our experiments. Voltages above $6 \mathrm{kV}$ caused the spray to become increasingly unstable, triggering an output of intermittent spurts of cells. Only a few droplets, if any, were directed towards the tissue culture plate. A jet spray was observed when higher cell numbers of $3 \times 10^{6} \%$ $\mathrm{ml}$ were used whereas and a plume spray was observed with lower cells numbers of $1 \times 10^{6} / \mathrm{ml}$ as seen in Fig. 2a, b. An unstable spray was observed at voltages above $6 \mathrm{kV}$ (Fig. 2c).

\subsection{Distribution of mBMSCs During Bio- Electrospray}

We next examined whether the mBMSCs were being delivered into the collecting tissue culture plate in even numbers during a spray period or whether cells were accumulating near the front or the back of the flow and hence arriving at an uneven rate into the collecting plate. This was achieved by collecting sequential fractions of cells as they emerged from the emitter. A suspension of mBMSCs at $3.5 \times 10^{6}$ cell $/ 300 \mu \mathrm{l}$ in c $\alpha$-MEM medium was sprayed at a flow rate of $5 \mu \mathrm{l} / \mathrm{min}$ and $100 \mu \mathrm{l}$. Three sequential fractions were collected in individual wells of a 24 well plate. Theoretically, if the flow of cells was continuous, $1.166 \times 10^{6}$ cells should be present in each fraction. It was found that over three separate experiments, fractions 1,2 and 3 contained $90 \pm 0.04,87 \pm 0.1$ and $90 \pm 0.02 \%$ of the theoretical value (Fig. 3). This indicates 
Fig. 4 Graph of Bio-

Electrosprayed mBMSCs

Viability. Viability of BES

mBMSCs was detected using both a EB/OA and b 7-AAD

viability staining solutions. Both stains are dye exclusion assays which identify nonviable cells by light microscope or by flow cytometry respectively. c Graph represents the \% of viable $\mathrm{BES}$ mBMSC and non-BES (n-3)

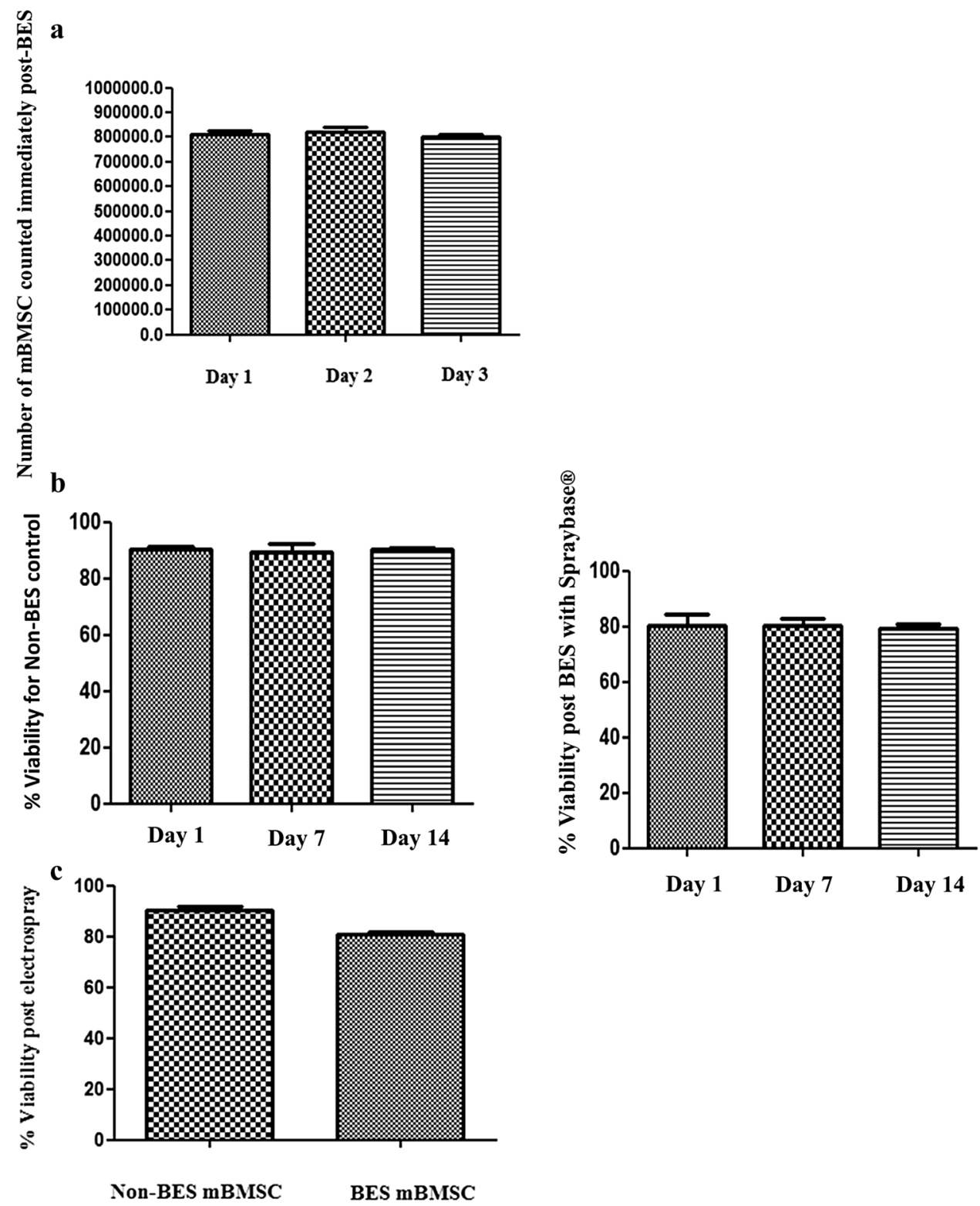

that the cells were being delivered in even numbers during the spray period.

\subsection{Viability of Bio-Electrosprayed mBMSCs}

Previous studies have shown that strong external electric fields, from 0.5 to $2.0 \mathrm{kV} / \mathrm{cm}$, can induce pore formation and cell membrane damage, leading to high levels of cell death [36-39]. Chen et al, further demonstrated that high voltages induce internal thermal damage to cells [39]. The viability of mBMSCs following BES using the optimized parameters was determined. It was found that approximately $80 \times 10^{4} \pm 1.7, \quad 82 \times 10^{4} \pm 1.9 \quad$ and $80 \times 10^{4} \pm 0.7 \%$ were viable mBMSCs when counted post BES. This indicated a high volume of mBMSCs were electrospraying into the wells (n-3). To further prove mBMSCs viability, a known dye exclusion assay (7-ADD) was carried out on both non-BES and BES cells. Analysis at day 1,7 , and 14 demonstrated that approximately $90 \pm 0.9 \%, 89 \pm 2.9 \%, 90 \pm 0.4 \%$ cells remained viable for non-BES, whereby $80 \pm 3.9 \%, 80 \pm 2.6 \%, 79 \pm 1.2 \%$ cells remained viable following BES (n-3). It was also shown that there was no significance between electrosprayed cells $80 \pm 1.3 \%$ and non-BES mBMSCs $90 \pm 1.5 \%$ as demonstrated in Fig. 4a-c. 
Table 3 mBMSC Surface marker expression

\begin{tabular}{lr}
\hline Bio-electrosprayed surface markers & \pm \\
\hline Sca-1 & + \\
CD 44 & + \\
CD 73 & + \\
CD 90 & + \\
CD 105 & + \\
CD 106 & + \\
MHC class I & + \\
CD $11 b$ & - \\
CD 34 & - \\
CD 45 & - \\
CD 86 & - \\
MHC class II & - \\
CD 117 & -
\end{tabular}

Table demonstrating 13 known surface markers that are either positively or negatively expressed by mBMSCs, according to guidelines outlined by the International Society for Cellular Therapy (ISCT)

\subsection{Characterization of mBMSCs Pre- and Post- BES}

In order to confirm the phenotype of the mBMSCs population used in these studies, marker expression was examined for both non-BES and BES cells. Expression of thirteen characteristic MSC cell surface markers, as described by the International Society for Cellular Therapy (ISCT), were examined using flow cytometry (Table 3) [40]. Expression of Sca-1, CD44, CD73, CD90, CD105, CD106 with minimum MHC Class I expression, and the absence of CD11b, the lymphocyte maker CD34, haematopoietic marker $\mathrm{CD} 45$, co-stimulatory marker CD86, MHC Class II, and CD117 demonstrated the expected mBMSCs phenotype for both non-BES and BES cells. This was illustrated when the cell surface marker represented by the red line using flow cytometry, "shifted" to the right of its corresponding isotype or control (black line), indicating a positive expression was determined. However, if the red line remained either within the black line or "shifted" to the left, this indicated a negative expression and thus the absence of the marker. Comparing the non-BES and BES mBMSCs percentage surface marker shift, the results demonstrated that the BES process did not alter cell surface marker expression in the electrosprayed cells shown in Fig. 5a and b.

\subsection{Multilineage Differentiation of Bio- Electrosprayed mBMSCs}

Having demonstrated that acceptable levels of cell viability were attained with our optimised BES protocol, we then examined the effect of the protocol on MSC function. Three key characteristics were analysed: multilineage differentiation ability, suppression of T-cell proliferation and pro-reparative capability. By definition, MSCs must show multilineage differentiation capability, for example towards osteoblast, adipocyte and chondrocyte lineages [1]. We therefore examined the ability of mBMSCs to differentiate towards osteoblast, adipocyte and chondrocyte lineages. The ability of differentiation capability of mBMSCs that had been subjected to BES was also examined to determine if the electrospray process adversely affected this capability.

For osteoblast, adipocyte and chondrocyte differentiation, cells were cultured under specific differentiation conditions for 21 days. Cells were then analysed for expression of differentiation-specific features. Osteoblasts were stained with Alizarin Red S Stain to visualise calcium deposits, adipocytes were stained with Oil Red O Isopropanol to visualise fat deposits and RNA was extracted from chondrocytes for detection of expression of markers genes. Differentiation for the three linages was observed either by micrographs (osteoblasts and adipocytes) or by marker expression as seen in chondrocyte differentiation, for both non-BES mBMSCs and BES mBMSCs (Fig. 6). Similar patterns of calcium and fat deposits were present in non-BES mBMSCs and BES mBMSCs differentiated cells. Similarly, expression of aggrecan and collagen IIa was increased in both non-BES mBMSCs and BES mBMSCs. However, due to a small percentage of cell loss (approx. $20 \%$ ) possibly from cell death, sticking to the inside of the syringe or from harvesting the cells post-electrospray, the sample for chondrocyte differentiation was smaller than the non-BES mBMSCs. This is demonstrated in the GAPDH analysis. Given the differences in GAPDH expression and band intensity for aggrecan and collagen IIa by rt-PCR, the expression of each chondrocyte marker was normalised to their relative GAPDH result. This demonstrated mBMSCs cultured in non-differentiating chondrocyte medium did not express either aggrecan or collagen IIa for both nonBES and BES cells. However, mBMSCs exposed to the chondrocyte differentiation medium showed an increase in both aggrecan and collagen IIa for the non-BES and BES mBMSCs (Fig. 6c). It's suggested that the expression levels are convincing as both aggrecan and collagen IIa have shown an increase in expression, indicating chondrocyte differentiation occurred, and that the differentiation capability of mBMSC is not adversely affected by this BES methodology. 

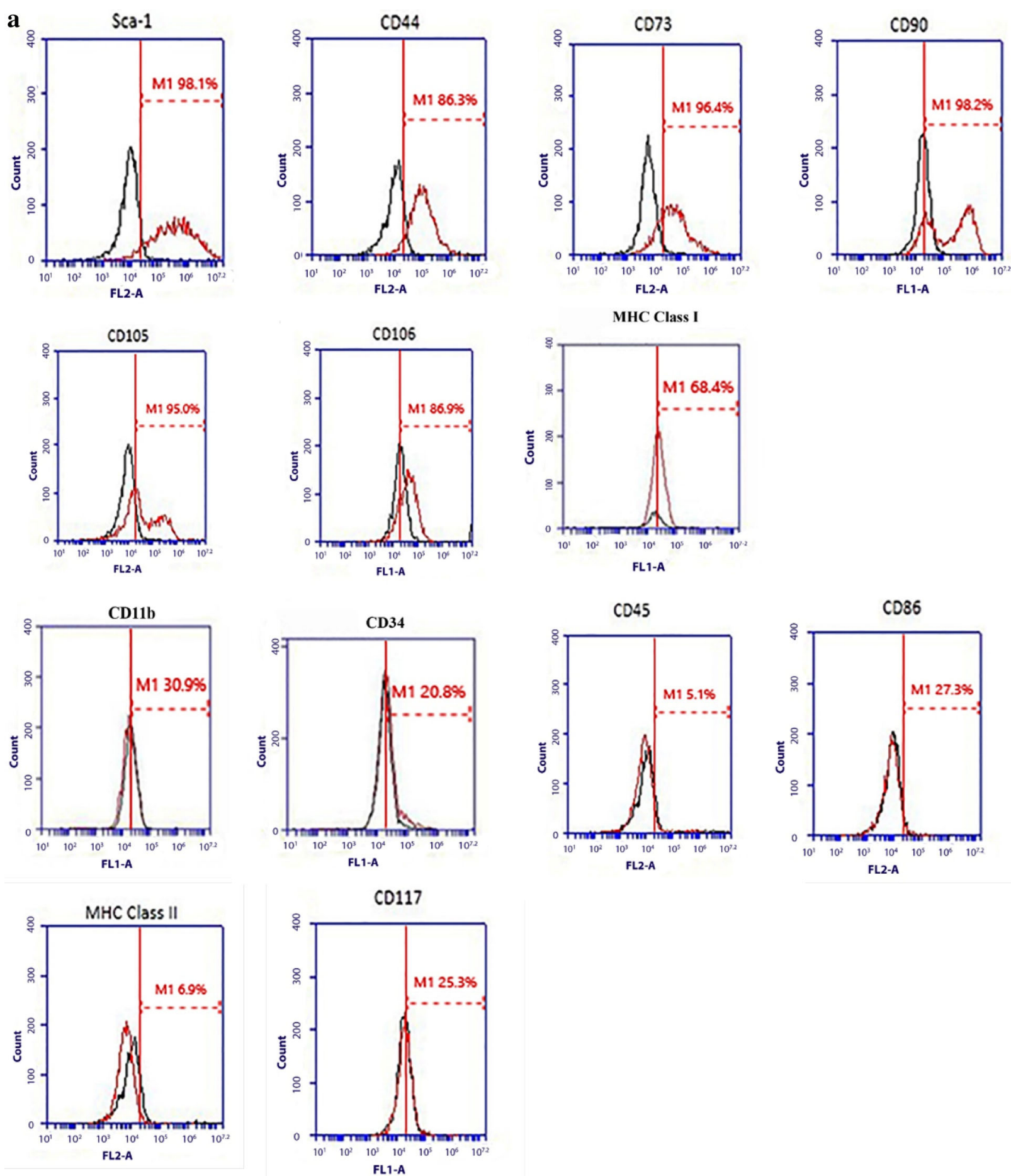

Fig. 5 Surface Marker Expression for Bio-Electrosprayed mBMSCs. mBMSCs are characterised by either the expression or absence of known surface markers. Flow cytometry showed that the a non-BES mBMSCs, represented by pipette cells, and b BES mBMSCs behaved similarly as they positively and negatively expressed 13 markers associated with mBMSCs. This was illustrated when the cell surface marker represented by the red line "shifted" to the right of its corresponding isotype or control (black line), indicating a positive expression was determined. However, if the red line remained either within the black line or "shifted" to the left, this indicated a negative expression and thus the absence of the marker 

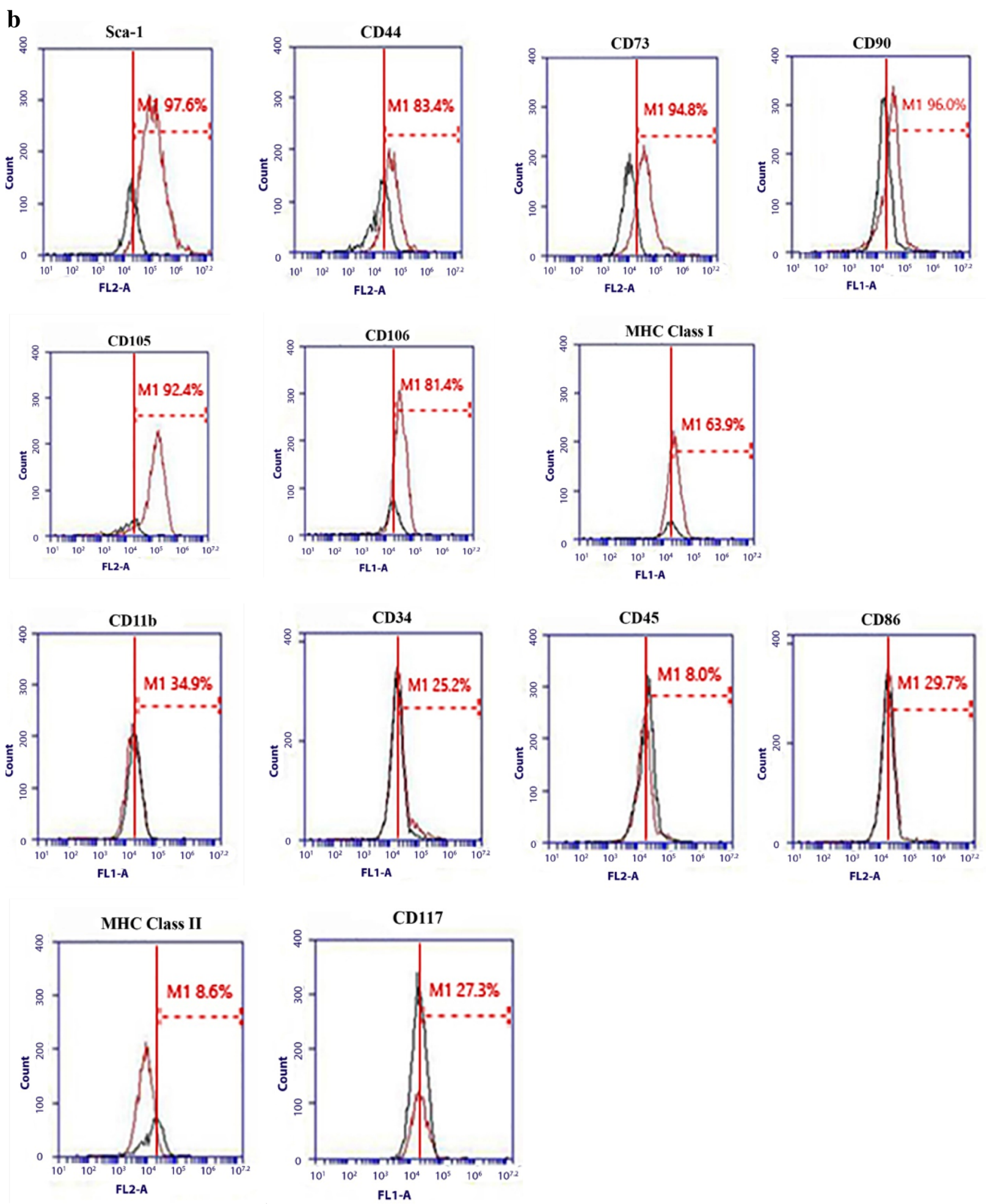

Fig. 5 continued 
$\mathbf{a}$

Undifferentiated Control

Osteoblast Differentiation

b

Undifferentiated Control

Adipocyte Differentiation
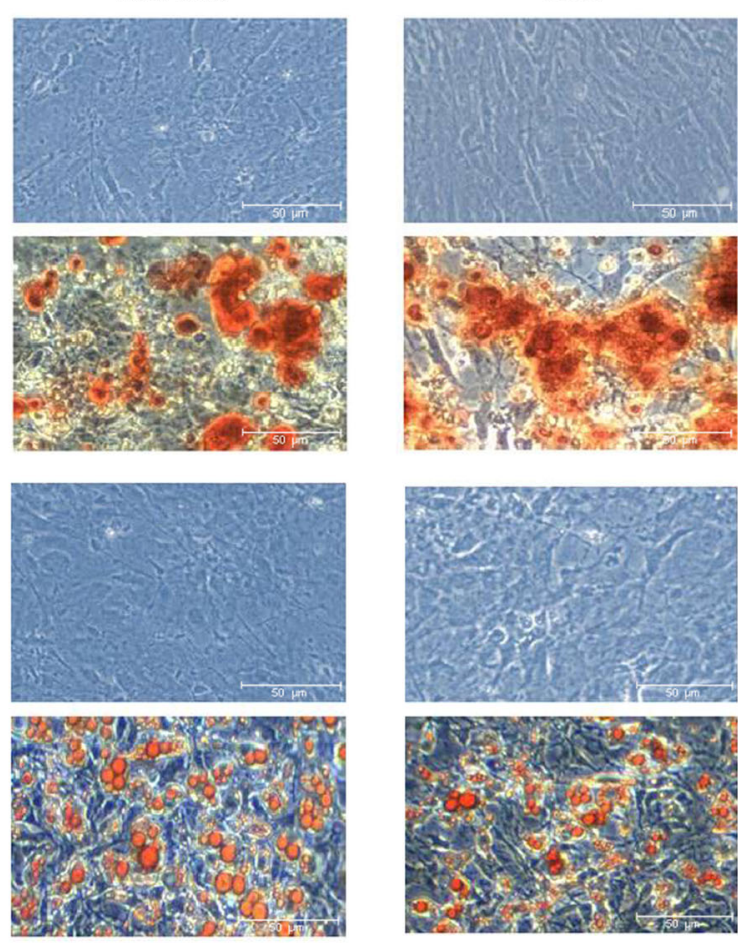

c

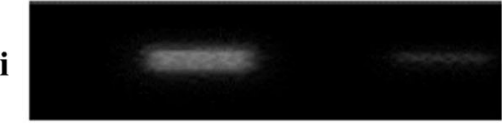

ii

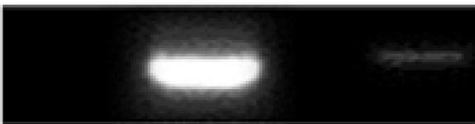

Collagen IIa

iii
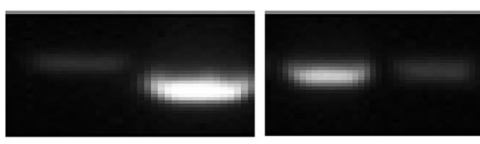

mBMSC Chondr mBMSC Chondr Non-BES Differn BES Differn Non-BES
GAPDH

Aggrecan

GAPDH
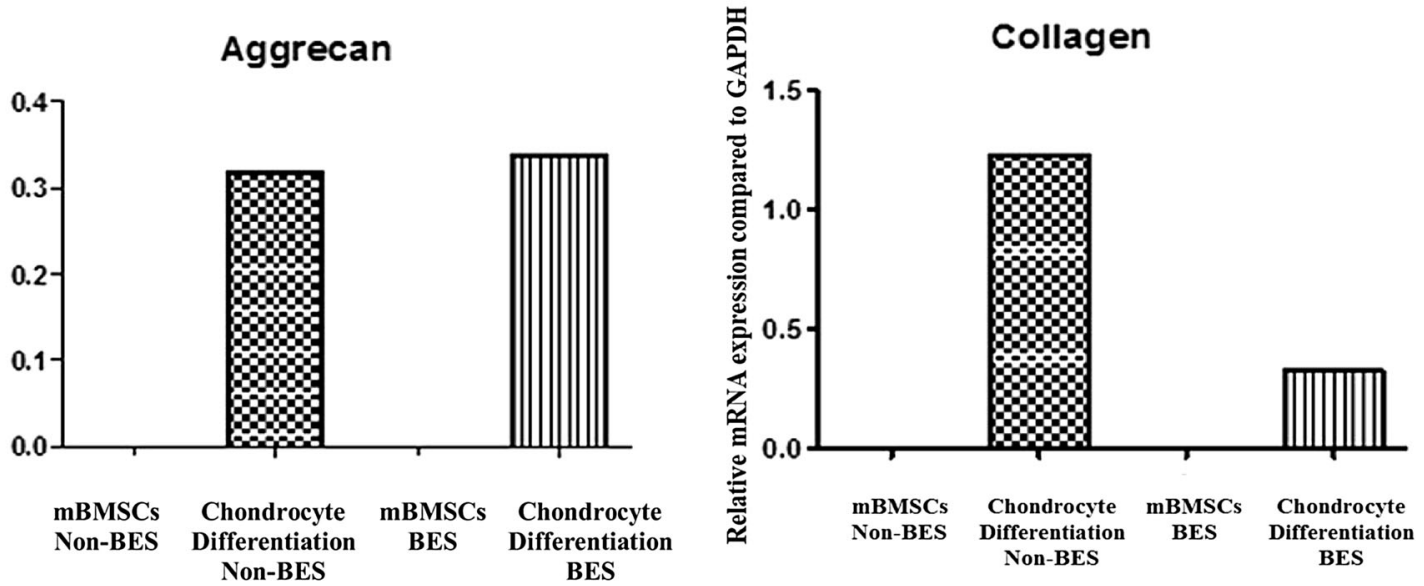
4Fig. 6 Osteogenic, Adipogenic and Chondrogenic Differeniation of Bio-Electrosprayed mBMSCs. a shows non-BES and BES mBMSC which differentiated into bone after 21 days with controls at $\times 10$ magnification. This is illustrated by reddish/orange calcium deposits apparent when stained with Alizarin Red. b shows non-BES and BES mBMSCs differentiated into fat after 21 days with controls at $\times 10$ magnification. This is shown by orange fat deposits when the differentiated mBMSCs were stained with Oil Red O. Controls remained undifferentiated when stained with either Alizarin Red (a) or with Oil Red O (b). c PCR showing expression levels of aggrecan and collagen IIa, both of which are present in cartilage, after 21 days. (i) Shows expression for aggrecan for both non-BES and BES mBMSCs. (ii) Shows expression levels for collagen IIa for both non-BES and BES mBMSCs, and (iii) shows the expression of GAPDH, a house keeping gene used as a control for comparing of gene expression data. The presence of these genes indicates that the BES mBMSCs retained their chondrogenic differentiation potential. Controls remained undifferentiated, this is shown by the lack of gene expression. Given the differences in GAPDH expression and band intensity for aggrecan and collagen IIa by rt-PCR, the expression of each chondrocyte marker was normalised to their relative GAPDH result as demonstrated by the mRNA expression graphs

\subsection{Suppression of T-cell Proliferation by Bio- Electrosprayed mBMSCs}

BES mBMSCs were examined for their capacity to suppress both autologous and allogeneic proliferation T-cells $[41,42]$. MHC mis-matched splenocytes from BALB/c (D1) and C57BL/6 (D2) were cultured with both non-BES and BES mBMSCs, and analysed by CFSE uptake into replicating DNA after $72 \mathrm{~h}$. In a second study, identical parameters where implemented with the inclusion of Concovalin A (ConA) at day 0 . This is a mitogen known for inducing T-cell proliferation, and was used as a positive control [43]. Although some proliferation was identified for both studies, significant immunosuppressive capacity for both non-BES and BES mBMSCs was demonstrated for alloantigen and mitogen driven proliferation. Statistical significance was determined by ANOVA analysis $(* * \mathrm{p}<0.01),(* * * \mathrm{p}<0.001)$. Results from these studies indicated proliferation of T-cells was significantly reduced in the presence of both non-BES mBMSCs and BES mBMSCs, with 4-fold proliferation reduction rates respectively compared to controls as demonstrated Fig. 7ac.

\subsection{Wound Closure Assay}

Studies have shown that MSCs migrate to injury sites and promote repair through the production of trophic factors, including growth factors, cytokines, and antioxidants $[44,45]$. We therefore investigated whether the jetting process would adversely affect the pro-reparative properties of mBMSCs. For this study, an in vitro wound closure assay was employed and wound closure was examined using the A549 lung epithelial cell line. Both non-BES and BES mBMSCs induced significantly higher rates of wound closure compared to controls. Wound sizes of $16 \pm 2.2 \%$ remained for the non-BES mBMSCs and $15 \pm 3.1 \%$ for the BES mBMSCs compared to control wounds. There was no significant difference between the wounds remaining in wells treated with non-BES or BES mBMSCs (Fig. 8). Comparable results were obtained when the BEAS-2B lung cell line was used as an alternative to A549 in the wound closure assay (results not shown).

\section{Discussion}

MSC therapeutic approaches currently involve administration via I.V. injection for cell therapies or scaffold transplantation for tissue engineering and regenerative medicine purposes. However, major challenges with MSC entrapment and difficulties with cell-scaffold interactions have hampered progress with these approaches. Bio-electrospray-mediated delivery where MSCs can be delivered directly to the target area has potentially several advantages compared with injection and scaffolds. The aim of this study was to develop a bio-electrospray methodology that could be ultimately compatible with in vivo delivery of MSCs. In previous studies by other groups, high voltages have been required to generate and maintain a continuous, stable electrospray of cells. High voltages are not acceptable therapeutically however because of both patient safety concerns and reduced MSCs viability and function. Furthermore, these groups have also used "in-house" electrospray apparatuses which means that reproducibility of experiments between labs is extremely difficult, if not impossible. In this study, we optimized electrospray parameters using a commercially available instrument, Spraybase ${ }^{\circledR}$. Since a large number of parameters affect the Taylor cone and plume/jet, including environmental conditions such as humidity and temperature, the spray mode can vary considerable between experiments, and even within experiments. The presence of a laser/camera visualisation system enabled close monitoring of the Taylor cone and plume/jet thus allowing the user to fine tune applied voltage for an optimal spray mode.

As discussed above, low voltages are essential during bio-electrospraying, both for cell viability and functions and for translation in vivo. Studies from other groups have demonstrated that strong external electric fields can negatively impact cells [36-39]. Using Spraybase ${ }^{\circledR}$ we successfully optimised parameters to enable BES of mBMSCs at low voltages, ranging between 3 and $6 \mathrm{kV}$. In agreement with a previous study by Odenwalder et al., we found that the ring-shape ground electrode encouraged the spray 
Fig. 7 Suppression of T-Cell Proliferation by Bio-

Electrosprayed mBMSCs.

a Represents the induction of

T-cell proliferation when two mismatched donor mice, i.e., BALB/c (D1) and C57/BL6 ((D2) were co-cultured together. The graph clearly shows significant T-cell suppression when BES mBMSCs are introduced indicating the mBMSCs are affecting T-cell proliferation. Graph (b) shows similar results. The introduction of mitogen Con A suggests a higher output of T-cell proliferation, yet when BES mBMSCs are present, T-cells are suppressed 4-fold (c)
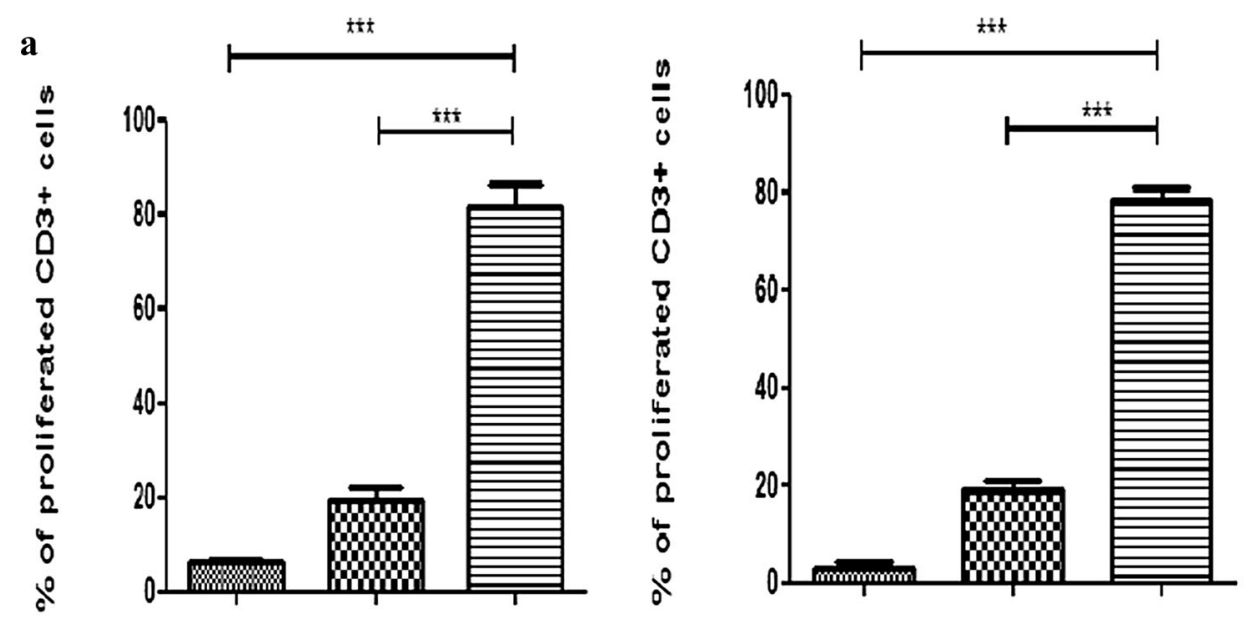

Non-EES N/SC -

Conor 1

Comor 2
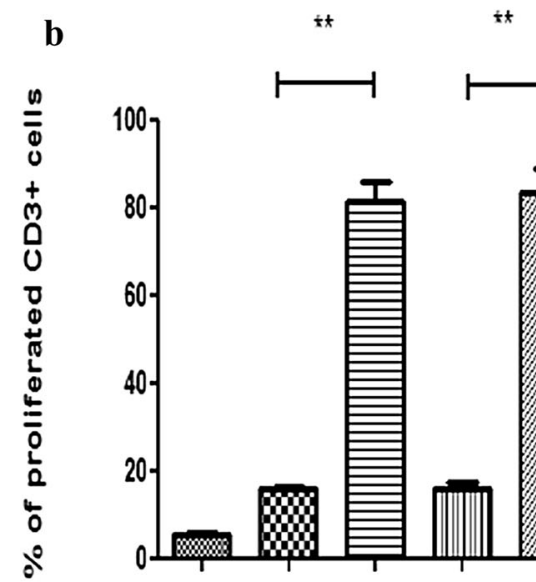

b

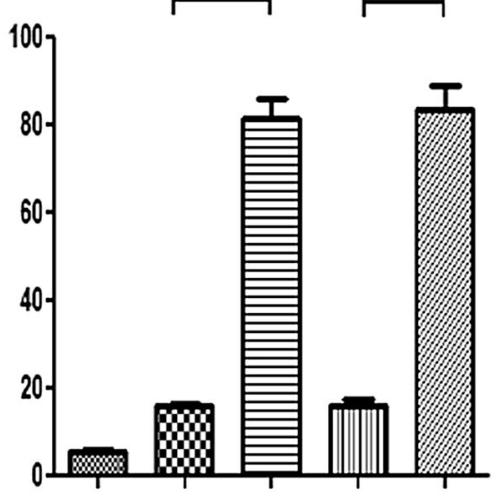

Non-BES MSC +

Donor

Donor 2

ConA
BES ISC +

Donor 1

Dors 2

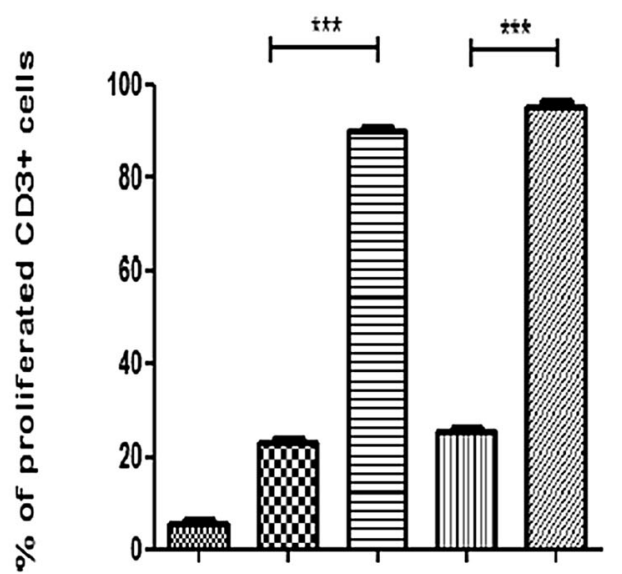

BES MSC

Doner !

Donor 2

$\operatorname{Con} A$

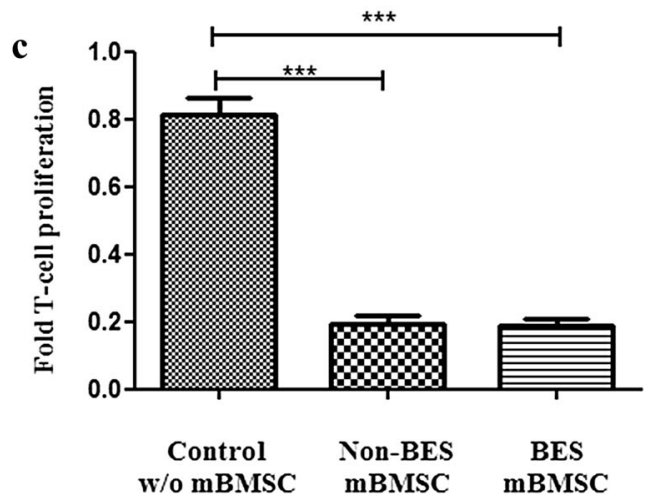


Fig. 8 Wound Closure using BES mBMSCs Pro-Reparative Properties on A549 Cells. Wound was created and observed over $24 \mathrm{~h}$ for 4 conditions described in 2.8 (n3). a Crystal violet staining of A549 cells show little to no closure to the wells with controls. b Plates with transwells containing either non-BES or BES mBMSCs illustrate wound closure. After $20 \mathrm{~h}$, wound was fully closed in well containing BES mBMSCs). c Graph demonstrates how much of the A549 cell wound was closed by control ( $c \alpha-M E M$ medium only), in relation to closure with BES mBMSCs. It's indicated it's the presence of BES mBMSCs which closed the wound in comparison to the controls. Graph shows there are no significant difference between non-BES mBMSCs and BES mBMSCs, thus indicating mBMSCs still retain their reparative properties after electrospraying

$\mathbf{a}$

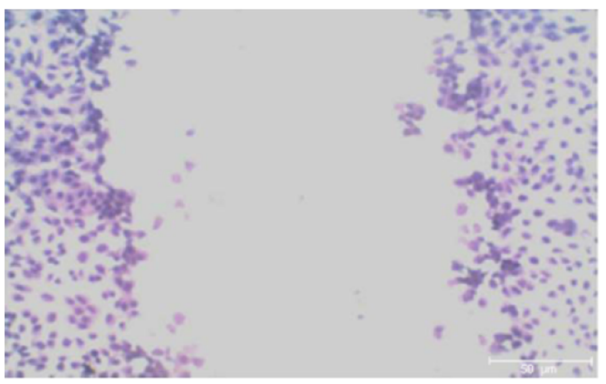

\section{ca-MEM \\ (control)}

$\mathbf{b}$

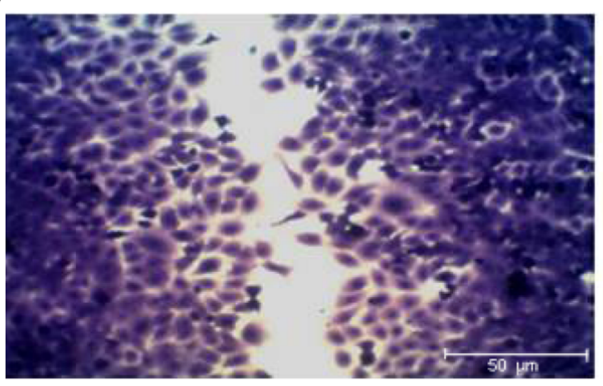

Non-BES mBMSC

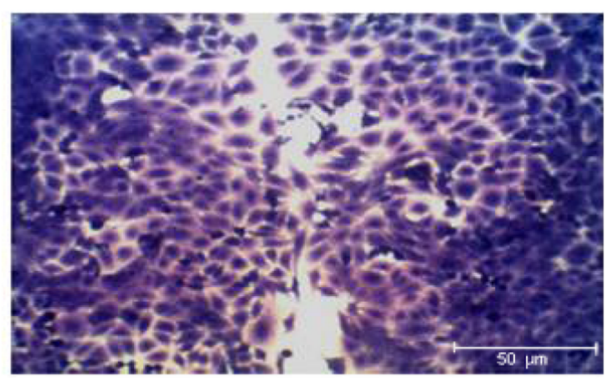

BES mBMSC

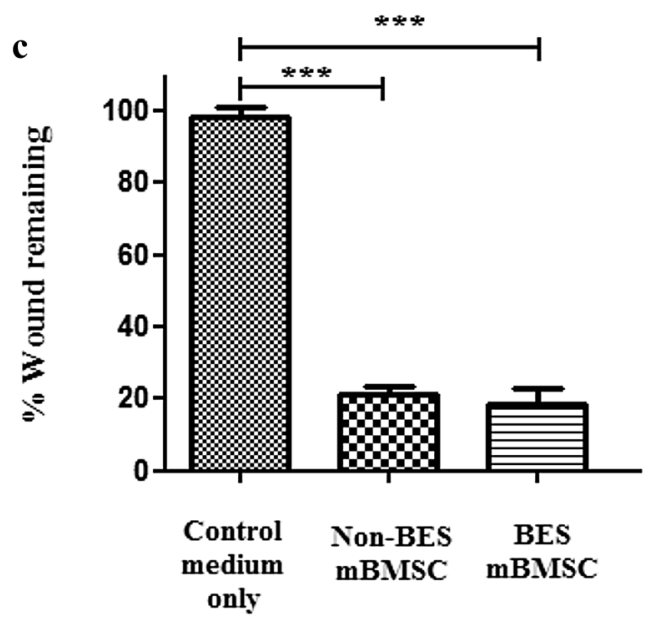

droplets to spread [32]. This design of electrode allowed cells to cover a relatively wide surface area whilst also making the spray more controllable. However, unlike the previous study, we successfully achieved a stable jet with a single needle configuration. Odenwalder et al. suggested that very high ion concentrations in combination with the low viscosity of their cell suspensions was the cause of jet instability in their experiments. As described by other groups $[24,28]$, we used solutions with high cell numbers to overcome the problem of low viscosity. The combination of the apparatus with high cell numbers and c $\alpha$-MEM

medium allowed us to reproducibly produce stable sprays and jets achieved at low voltages using a single extrusion needle configuration.

Successful electrospray of a number of primary cells, including various types of stem cells, has been reported previously [23-28]. However, a full phenotypic analysis of MSCs post BES has not yet been described [24]. Therefore, a key part of our study was the analysis of the effect of low voltage electrospraying on mBMSCs function. In addition to surface marker expression, multilineage differentiation, immunosuppressive effects and pro-reparative properties 
were evaluated following BES in vitro. These studies demonstrated that the jetting methodology did not adversely affect mBMSCs phenotype or function. To our knowledge this is the first time such an in-depth analysis of this methods effects on MSCs specifically, have been demonstrated. This suggests using BES method to electrospray MSCs to a given target site, has the potential as an alternative and translational tool for delivering these cells.

MSCs hold great promise as therapeutic entities and as such are receiving significant levels of attention. By 2016, MSC therapeutics was estimated to be worth of $\$ 8.8$ billion [46]. However, the challenges encountered with current delivery methods illustrate that new and innovative methods for MSCs administration are required. The recommended number of cells administrated in cell therapies is $1-2 \times 10^{6} \mathrm{MSC} / \mathrm{kg}$ [47]. However, due to the aforementioned problems a substantial number of these cells may be lost, giving limited success to the various therapies. Therefore, BES holds potential as a delivery method for MSCs, and other cell types, as it could enable direct delivery of known number of cells to a target area in vivo. This research is based on laboratory apparatus using a device designed for commercial lab based experimental studies. Theoretically, using this newly adapted apparatus and optimised parameters, MSC therapeutics could be used for any organ through keyhole surgery promoting advances towards cellular and tissue engineering purposes using a specially designed medical device. This is a critical step towards the translation of electrospray technologies for delivery of cells for therapeutic purposes in vivo.

Acknowledgements This work was funded by the HEA under PRTLI5 and by EU FP7 Marie Cuire IAPP and is being co-funded by the Irish Government and the EU under Ireland's Structural Funds Programmes 2007-2013: Investing in your future.

Funding SAC funded under SFI Grant 13/IA/1840.

\section{References}

1. Pittenger, M. F., Mackay, A. M., Beck, S. C., Jaiswal, R. K., Douglas, R., Mosca, J. D., et al. (1999). Multilineage potential of adult human mesenchymal stem cells. Science, 284(5411), 143-147.

2. Ding, Y. C., Xu, D. M., Feng, G., Bushell, A., Muschel, R. J., \& Wood, K. J. (2009). Mesenchymal stem cells prevent the rejection of fully allogenic islet grafts by the immunosuppressive activity of matrix metalloproteinase-2 and -9. Diabetes, 58(8), 1797-1806.

3. Aggarwal, S., \& Pittenger, M. F. (2005). Human mesenchymal stem cells modulate allogeneic immune cell responses. Blood, 105(4), 1815-1822.

4. Eggenhofer, E., Benseler, V., Kroemer, A., Popp, F. C., Geissler, E. K., Schlitt, H. J., et al. (2012). Mesenchymal stem cells are short-lived and do not migrate beyond the lungs after intravenous infusion. Frontiers in Immunology. doi:10.3389/fimmu.2012. 00297.eCollection.

5. English, K., French, A., \& Wood, K. J. (2010). Mesenchymal stromal cells: Facilitators of successful transplantation? Cell Stem Cell, 7(4), 431-442.

6. McKernan, R., McNeish, J., \& Smith, D. (2010). Pharma's developing interest in stem cells. Cell Stem Cell, 6(6), 517-520.

7. Schu, S., Nosov, M., O’Flynn, L., Shaw, G., Treacy, O., Barry, F., et al. (2012). Immunogenicity of allogeneic mesenchymal stem cells. Journal of Cellular and Molecular Medicine, 16(9), 2094-2103.

8. Nystedt, J., Anderson, H., Tikkanen, J., Pietilä, M., Hirvonen, T., Takalo, R., et al. (2013). Cell surface structures influence lung clearance rate of systemically infused mesenchymal stromal cells. Stem Cells, 31(2), 317-326.

9. Plock, J. A., Schnider, J. T., Schweizer, R., \& Gorantla, V. S. (2013). Are cultured mesenchymal stromal cells an option for immunomodulation in transplantation? Frontiers in Immunology. doi:10.3389/fimmu.2013.00041.eCollection.

10. Ji, W., Sun, Y., Yang, F., van den Beucken, J. J. J. P., Fan, M. W., Chen, Z., et al. (2011). Bioactive electrospun scaffolds delivering growth factors and genes for tissue engineering applications. Pharmaceutical Research, 28(6), 1259-1272.

11. Kai, D., Jin, G., Prabhakaran, M. P., \& Ramakrishna, S. (2013). Electrospun synthetic and natural nanofibers for regenerative medicine and stem cells. Biotechnology Journal, 8(1), 59-72.

12. Tamayol, A., Akbari, M., Annabi, N., Paul, A., Khademhosseini, A., \& Juncker, D. (2013). Fiber-based tissue engineering: Progress, challenges, and opportunities. Biotechnology Advances, 31(5), 669-687.

13. Shin, S. H., Purevdorj, O., Castano, O., Planell, J. A., \& Kim, H. W. (2012). A short review: Recent advances in electrospinning for bone tissue regeneration. Journal of Tissue Engineering. doi: $10.1177 / 2041731412443530$.

14. Agarwal, S., Wendorff, J. H., \& Greiner, A. (2008). Use of electrospinning technique for biomedical applications. Polymer, $49(26), 5603-5621$.

15. Lee, S. H., \& Shin, H. (2007). Matrices and scaffolds for delivery of bioactive molecules in bone and cartilage tissue engineering. Advanced Drug Delivery Reviews, 59(4-5), 339-359.

16. Huang, Z. M., Zhang, Y. Z., Kotaki, M., \& Ramakrishna, S. (2003). A review on polymer nanofibers by electrospinning and their applications in nanocomposites. Composites Science and Technology, 63(15), 2223-2253.

17. Rim, N. G., Shin, C. S., \& Shin, H. (2013). Current approaches to electrospun nanofibers for tissue engineering. Biomedical Materials, 8(1), 014102.

18. Taylor, G. (1964). Disintegration of water drops in an electric field. Proceedings of the Royal Society A, 280(1382), 383-397.

19. Jayasingh, S. N., Qureshi, A. N., \& Eagles, P. A. M. (2006). Electrohydrodynamic jet processing: An advanced electric-fielddriven jetting phenomenon for processing living cells. Small (Weinheim an der Bergstrasse, Germany), 2(2), 216-219.

20. Jayasinghe, S. N., Eagles, P. A. M., \& Qureshi, A. N. (2006). Electric field driven jetting: An emerging approach for processing living cells. Biotechnology Journal, 1(1), 86-94.

21. Jayasinghe, S. N. (2011). Bio-electrosprays: From bio-analytics to a generic tool for the health sciences. Analyst, 136(5), 878-890.

22. Salata, O. V. (2005). Tools of nanotechnology: Electrospray. Current Nanoscience, 1(1), 25-33.

23. Ng, K. E., Joly, P., Jayasinghe, S. N., Vernay, B., Knight, R., Barry, S. P., et al. (2011). Bio-electrospraying primary cardiac cells: In vitro tissue creation and functional study. Biotechnology Journal, 6(1), 86-95. 
24. Sahoo, S., Lee, W. C., Goh, J. C., \& Toh, S. L. (2010). Bioelectrospraying: A potentially safe technique for delivering progenitor cells. Biotechnology and Bioengineering, 106(4), 690-698.

25. Bartolovic, K., Mongkoldhumrongkul, N., Waddington, S. N., Jayasinghe, S. N., \& Howe, S. J. (2010). The differentiation and engraftment potential of mouse hematopoietic stem cells is maintained after bio-electrospray. Analyst, 135(1), 157-164.

26. Abeyewickreme, A., Kwok, A., McEwan, J. R., \& Jayasinghe, S. N. (2009). Bio-electrospraying embryonic stem cells: Interrogating cellular viability and pluripotency. Integrative Biology: Quantitative Biosciences From Nano to Macro, 1(3), 260-266.

27. Guan, J., Wang, F., Li, Z., Chen, J., Guo, X., Liao, J., et al. (2011). The stimulation of the cardiac differentiation of mesenchymal stem cells in tissue constructs that mimic myocardium structure and biomechanics. Biomaterials, 32(24), 5568-5580.

28. Ye, C., He, Z., Lin, Y., Zhang, Y., Tang, J., Sun, B., et al. (2014). Bio-electrospraying is a safe technology for delivering human adipose-derived stem cells. Biotechnology Letters, 37(2), 449-456.

29. Rulison, A. J., \& Flagan, R. C. (1994). Electrospray atomization of electrolytic solutions. Journal of Colloid Interface Science, 167(1), 135-145

30. Kim, G., Park, J., \& Han, H. (2006). Production of microsized PMMA droplets using electrospraying with various auxiliary fields. Journal of Colloid Interface Science, 299(2), 593-598.

31. Hartman, R. P. A., Brunner, D. J., Camelot, D. M. A., Marijnissen, J. C. M., \& Scarlett, B. (2000). Jet break-up in electrohydrodynamic atomization in the cone-jet mode. Journal of Aerosol Science, 31(1), 65-95.

32. Odenwalder, P., Irvine, S., McEwan, J. R., \& Jayasing, S. N. (2007). Bio-electrosprays: A novel electrified jetting methodology for the safe handling and deployment of primary living organisms. Biotechnology Journal, 2(5), 622-630.

33. Peister, A., Mellad, J. A., Larson, B. L., Hall, B. M., Gibson, L. F., \& Prockop, D. J. (2004). Adult stem cells from bone marrow (MSCs) isolated from different strains of inbred mice vary in surface epitopes, rates of proliferation, and differentiation potential. Blood, 103(5), 1662-1668.

34. English, K., Barry, F. P., Field-Corbett, C. P., \& Mahon, B. P. (2007). IFN-gamma and TNFalpha differentially regulate immunomodulation by murine mesenchymal stem cells. Immunology Letters, 110(2), 91-100.

35. Quah, B. J. C., \& Parish, C. (2012). New and improved methods for measuring lymphocyte proliferation in vitro and in vivo using
CFSE-like fluorescent dyes. Journal of Immunological Methods, 379(1-2), 1-14.

36. Ho, S. Y., \& Mittal, G. S. (1996). Electroporation of cell membranes: A review. Critical Reviews in Biotechnology, 16(4), 349-362.

37. DeBruin, K. A., \& Krassowska, W. (1999). Modeling electroporation in a single cell. I. Effects of field strength and rest potential. Biophysical Journal, 77(3), 1213-1224.

38. Gass, G. V., \& Chernomordik, L. V. (1990). Reversible largescale deformations in the membranes of electrically-treated cells: Electroinduced bleb formation. Biochimica et Biophysica Acta, 1023(1), 1-11.

39. Chen, W., Han, Y., Chen, Y., \& Xie, J. T. (1998). Field-induced electroconformational damages in cell membrane proteins: A new mechanism involved in electrical injury. Bioelectrochemistry and Bioenergetics, 47(2), 237-245.

40. Dominici, M., Le Blanc, K., Mueller, I., Slaper-Cortenbach, I., Marini, F. C., Krause, D. S., et al. (2006). Minimal criteria for defining multipotent mesenchymal stromal cells. The International Society for Cellular Therapy position statement. $C y$ totherapy, 8(4), 315-317.

41. Di Nicola, M., Carlo-Stella, C., Magni, M., Milanesi, M., Longoni, P. D., Matteucci, P., et al. (2002). Human bone marrow stromal cells suppress T-lymphocyte proliferation induced by cellular or nonspecific mitogenic stimuli. Blood, 99(10), 3838-3843.

42. Glennie, S., Soeir, I., Dyson, P. J., Lam, E. W., \& Dazzi, F. (2005). Bone marrow mesenchymal stem cells induce division arrest anergy of activated T cells. Blood, 105(7), 2821-2827.

43. Dwyer, J. M., \& Johnson, C. (1981). The use of concanavalin A to study the immunoregulation of human $\mathrm{T}$ cells. Clinical and Experimental Immunology, 46(2), 237-249.

44. Chen, L., Tredget, E. E., Wu, P. Y., \& Wu, Y. (2008). Paracrine factors of mesenchymal stem cells recruit macrophages and endothelial lineage cells and enhance wound healing. PLOS ONE. doi:10.1371/journal.pone.0001886.eJournal.

45. Karp, J. M., \& Leng Teo, G. S. (2009). Mesenchymal stem cell homing: The devil is in the details. Cell Stem Cell, 4(3), 206-216.

46. Syed, B. A., \& Evans, J. B. (2013). From the analyst's couch stem cell therapy market. Nature Reviews Drug Discovery, 12(1), 185-186.

47. Hayat, M. A. (2012). Therapeutic applications in disease and injury. Stem Cells and Cancer Stem Cells. doi:10.1007/978-94007-2993-3.eBook. 